\title{
Reversible Fabric Heats and Cools
}

\section{A new theory proposes a reversible fabric that could potentially keep a person warm when worn one way and cool when flipped inside out.}

\author{
By Michael Schirber
}

( ur bodies lose a large fraction of their heat by radiating it to the environment, but clothing designers rarely try to control this radiation. A new theory proposes a wearable, reversible fabric that would emit close to zero radiation from one side while emitting a large amount from the other [1]. Unlike previous reversible fabrics that were made from impermeable membranes, this new fabric would be composed of microfibers, which would be more comfortable to wear. The thin fabric could be worn one way to keep warm in a cool room and flipped inside out to keep cool in a warm room. Energy-saving thermostat settings could then be used without

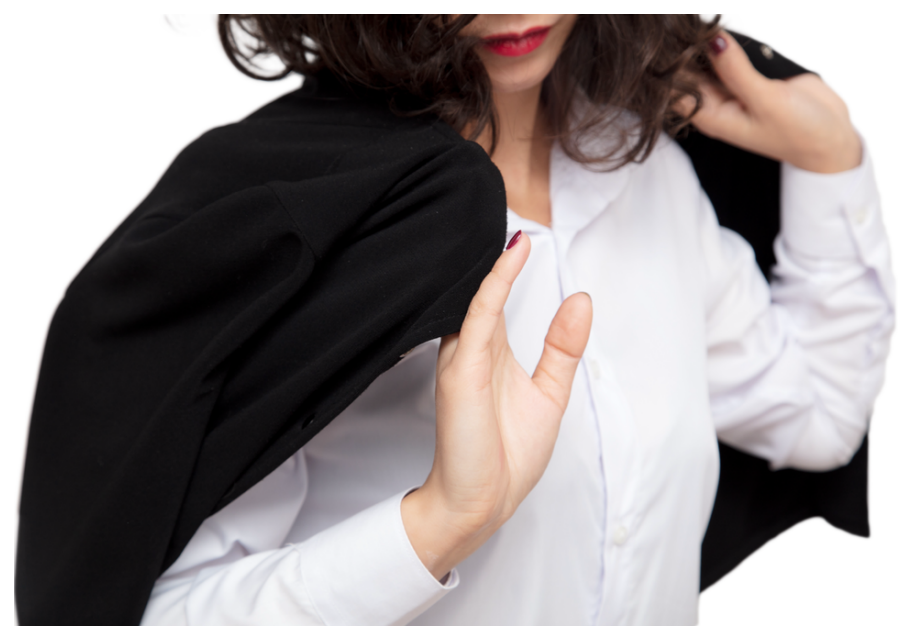

Taking both sides. New theoretical work explores a reversible fabric design that could trap radiative heat on one side, while letting it escape more easily on the other. So the material could work in both warm and cool environments, just by flipping it around.

Credit: schankz/stock.adobe.com causing discomfort.

At rest in an indoor environment, the body's emission of infrared radiation accounts for more than $50 \%$ of its total heat loss. The remaining amount comes from conduction (heat traveling from the skin to something in direct contact, such as clothing) and convection (heat transported away by air movement near the skin). A person can add layers of, say, cotton fabric to slow conduction or convection, but changing the radiative loss requires changing the ability of the outer surface of one's clothes to radiate.

Photonic materials offer researchers a way to tailor the radiation from an object (see Focus: Emitting Photons Is One Way to Be Cool). Recent work has explored so-called Janus textiles, which have different infrared "colors" on each side and thus different emission properties [2]. However, these materials have usually been membranes, which can be uncomfortable for the wearer because they trap air and humidity near the skin. So, Ph.D. student Muluneh Abebe from the University of Mons in Belgium and his colleagues have developed a theory for a Janus textile made of fibers knitted together in a way that allows airflow between them.

The design that Abebe and his colleagues came up with has metallic fibers on one side and dielectric fibers on the other. To characterize the fabric, the researchers calculated the transmission, reflection, and absorption of radiation impinging on the material. These radiative processes are time reversible, implying that the capacity to absorb radiation on a particular side is equivalent to the capacity to emit radiation from that same side. "Whatever heat is absorbed inside the material will 


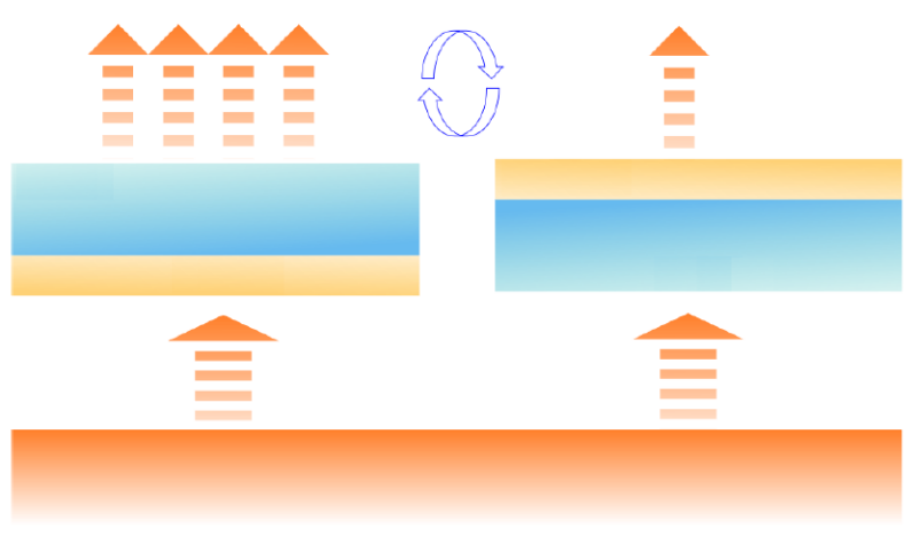

A thermal flip. Heat from the skin (orange layer) radiates or conducts into the Janus fabric, where it is emitted to the environment in a way that depends on the fabric's orientation. When the high emissivity dielectric fibers (blue) are facing outward, the amount of radiation is almost equivalent to bare skin, and the wearer feels cool. When flipped around, the low emissivity metallic fibers (gold) face outward, and the amount of radiation is nearly zero, so the wearer feels warm.

Credit: M. G. Abebe et al. [1]

be completely emitted from the surface in the reverse process," Abebe explains. The capacity to emit is characterized by the emissivity, which goes from 0 for a non-emitter to 1 for a perfect blackbody.

Metals are reflective, so they absorb little light, and thus the metallic fibers should have low emissivity. The team imagined two layers of metallic, micrometer-wide fibers arranged in a crossed pattern. Calculations of the emissivity from the metallic side gave a value of just 0.02 . By contrast, dielectric materials are highly absorbing and should be highly emissive. The researchers found high emissivity at the appropriate infrared wavelengths when the dielectric fibers were made of silicon carbide, had radii of around 1 micrometer, and had fiber separations of 3 micrometers. With these parameters, they calculated the emissivity of the dielectric side to be 0.74 .
The team then inserted these emissivities into a thermal model that determined the heat loss. They found that a person wearing the fabric with the metal side outward could feel comfortable in a cool room with the thermostat set to $11^{\circ} \mathrm{C}$ $\left(52^{\circ} \mathrm{F}\right)$. Even though the fabric would be only about 20 micrometers thick, its warming effect would be equivalent to wearing a moderately thick jacket, Abebe says. If the person then went to a warm environment, they could flip the fabric around and remain comfortable up to $25^{\circ} \mathrm{C}\left(77^{\circ} \mathrm{F}\right)$. So, with this thin garment available, less energy could be spent heating or cooling an entire office or home. The predicted "comfort zone" for this fabric-based Janus textile is slightly wider than that of membrane-based ones that have been measured $\left(14-24^{\circ} \mathrm{C}\right)[2]$.

Abebe and his colleagues are working with experimentalists to develop a prototype of their design. Metallic clothing might sound uncomfortably stiff, but Abebe says that the thinness of the fibers makes them sufficiently flexible.

Photonics expert Qiang Li from Zhejiang University in China says that there are good reasons to choose silicon carbide for the dielectric, as it has good strength and chemical stability. But practical issues stand in the way. "The fabrication of metal fiber and silicon carbide fiber is challenging, and the cost for mass manufacturing can be high," Li says. However, he says that if they can be made, fiber-based Janus textiles would offer good air circulation and personal comfort.

Michael Schirber is a Corresponding Editor for Physics based in Lyon, France.

\section{REFERENCES}

1. M. G. Abebe et al., "Janus-yarn fabric for dual-mode radiative heat management," Phys. Rev. Applied 16, 054013 (2021).

2. P.-C. Hsu et al., "A dual-mode textile for human body radiative heating and cooling," Sci. Adv. 3 (2017). 